\title{
Magnetic Behaviour of Non-interacting Colloidal Iron Oxide Nanoparticles in Physiological Solutions
}

\author{
S. Haracz ${ }^{1}$, B. Mróz ${ }^{2}$, J. D. Rybka ${ }^{1}$ and M. Giersig ${ }^{1,3 *}$ \\ ${ }^{1}$ Chemical Physics, Faculty of Chemistry, Adam Mickiewicz University, Umultowska 89B, \\ 61-614 Poznań, Poland \\ ${ }^{2}$ Crystal Physics Division, Faculty of Physics, Adam Mickiewicz University, Umultowska 85, \\ 61-614, Poznań, Poland \\ ${ }^{3}$ Freie University Berlin, Experimental Physics, Arnimalle 14, 14195 Berlin, Germany \\ Email: giersig@physik.fu-berlin.de, phone: +49 3083851404 fax: +49 3083856081
}

\begin{abstract}
Magnetic properties of colloidal nanoparticles (NPs) depend on various parameters, such as size, size distribution, interparticle distance, shape, condition of synthesis and stabilizing surfactants. Nowadays, those magnetic nanoparticles (MNPs) are preferably produced in hydrophobic organic solvents, while biomedical applications need hydrophilic properties. Thus, a major challenge is the hydrophilization of the particles, while avoiding destabilization and aggregation. Here we present magnetic characteristics of non-interacting, highly crystalline iron oxide NPs in physiological solutions that are coated with modified polyacrylic acid. The magnetic analysis comprised both static and dynamic magnetic behaviour of $4 \mathrm{~nm}$ nanoparticles. The nanoparticles have been further characterized by transmission electron microscopy (TEM) and energy dispersive X-ray spectroscopy (EDX).
\end{abstract}

\section{KEYWORDS}

Iron Oxide Nanoparticles; Superparamagnetism; SQUID; Bio-Application

\section{Introduction}

Recently, magnetic nanoparticles (NPs) have been widely investigated due to their potential in multiple biomedical applications, such as drug delivery, hyperthermia, or cell separation. The quality of highly-performed NPs of well-defined magnetic properties, i.e. magnetization, saturation and susceptibility, strongly depends on size, shape, and crystallinity of the particles[1]. In the last years new methods have been developed to improve both the magnetic properties and the stability of NPs in physiological solutions. Monodisperse iron oxide NPs are mostly prepared by thermal decomposition in organic solvents [2,3], while a biomedical application of superparamagnetic NPs requires $(i)$ stabilization in biological surroundings, i.e. pH 7.4 and physiological salt concentration, and (ii) surface-functionalization for further modifications, such as targeting with antibodies and/or passivation to avoid unspecific bindings. The most major problem of hydrophilization of hydrophobic NPs that has been published during many years is aggregation, i.e. missing long term stability. There are several approaches in phase transfer of hydrophobic iron oxide NPs into water and biofluids: 
manipulation by exploiting electrostatic interactions [4], coating with amphiphilic polymer [5], embedding in recombinant lipoproteins [6], or other surface modifications [2,7]. However, this problem is still challenging.

The identification of iron oxide type is important. Magnetite is ferrimagnetic below $860 \mathrm{~K}$, with a saturation magnetization value of $84 \mathrm{emu} / \mathrm{g}$. The net magnetization reflects the $\mathrm{Fe} 2+$ ions in the octahedral sites, whereas in maghemite the net magnetic moment originates from $\mathrm{Fe} 3+$ ions, with a magnetic order-disorder transition temperature close to $1020 \mathrm{~K}$ and a bulk saturation magnetization of $74 \mathrm{emu} / \mathrm{g}$. The results reveal that the NPs consist of a mixture of $\mathrm{Fe} 2 \mathrm{O} 3$ and $\mathrm{Fe} 3 \mathrm{O} 4$. Relative percentage ratios of each phase in the samples can be expressed in the form of $(\mathrm{gFe} 2 \mathrm{O} 3) 1 x(\mathrm{Fe} 3 \mathrm{O} 4) x$ with the $x=0.3$ for the $5 \mathrm{~nm}$ particles $[8,9,10,11]$.

In this paper we present a detailed study of long term stable, non-interacting iron oxide NPs in a buffer solution.

\section{Materials and methods}

\subsection{Iron Oxide Synthesis}

Iron oxide NPs were prepared by thermal decomposition of iron pentacarbonyl $(1 \mathrm{mmol})$ in the presence of oleic acid $(1 \mathrm{mmol})$ and $10 \mathrm{ml}$ of 1-octadecene [2,3]. The mixture, submerged in nitrogen and stirred, was then heated up to reflux $\left(320^{\circ} \mathrm{C}\right)$ for one hour. Afterwards the resulting solution was cooled down to room temperature. The solution was treated with excess ethanol and separated by centrifugation and redispersed in chloroform.

\subsection{Polymer Synthesis}

Modified-polyacrylic acid (mPAA, $1 \mathrm{~g}$ ) was synthesized according to Insin (PhD-thesis, Massachusetts Institute of Technology) [12]. Briefly, polyacrylic acid was dissolved in $10 \mathrm{ml}$ of DMF (dimethylformamide) and then N-octyl amine was added. The reaction mixture was stirred for 2 hours before the addition of 1-ethyl-3-(3-dimethylaminopropyl) carbodiimide (EDC, $1.06 \mathrm{~g}$ ). Then, the mixture was stirred at room temperature for 20 hours. Afterwards, DMF was removed under reduced pressure and $2 \mathrm{ml}$ of water and $1 \mathrm{~g}$ of tetramethylammonium hydroxide were added and stirred for $2 \mathrm{~h}$. Finally, hydrochloric acid was added in order to re-precipitate the mPAA and then the supernatant was removed. The purified mPAA was kept as is or dissolved in ethylacetate.

\subsection{Coating Process of Iron Oxide Nanoparticles}

Magnetic NPs (5 mg, incl. surfactant) were mixed with mPAA in chloroform for $12 \mathrm{~h}$. An extent of chloroform was slowly reduced by pressure, and then PBS (phosphate buffer saline) was added dropwise under ultrasonification of the particle solution.

\section{4 mPAA modification}

MNPs were subsequently coated with mPAA in PBS buffer, mixed with EDC [13] for $2 \mathrm{~h}$ before polyethyleneglycol-bis(3-amino-propyl) was added in order to functionalize the nanoparticles with hydrophilic amino groups. The solution was then stirred for $12 \mathrm{~h}$. 


\section{Results and Discussion}

\subsection{Raman Spectroscopy}

Raman spectroscopy (RenishawinVia, with a $514 \mathrm{~nm}$ laser) was exploited to confirm the structure of modified-polyacrylic acid before the coating of the NPs. Figure 1 shows the Raman spectra of modified polyacrylic acid. The Raman-measurements showed two significant regions. The first one (a) is located between $400 \mathrm{~cm}^{-1}$ to $1500 \mathrm{~cm}^{-1}$ and shows four peaks corresponding to the structure of the polymer. One band at $580 \mathrm{~cm}^{-1}$ hat is correlated with the NCO groups absorption, a band at $753 \mathrm{~cm}^{-1}$ that correlates with the COO groups, and two other coming from oscillations of alkyl groups. The other appointed range (b) stretched from $2600 \mathrm{~cm}^{-1}$ to $3200 \mathrm{~cm}^{-1}$ and was entailed by oscillation of alkyl and amino groups of the polymer. According to literature [14,15] two peaks of $580 \mathrm{~cm}^{-1}$ and $3030 \mathrm{~cm}^{-1}$ are absent in purified polyacrylic acid. Therefore an amine bond between polyacrylic acid and octylamine can be suggested.
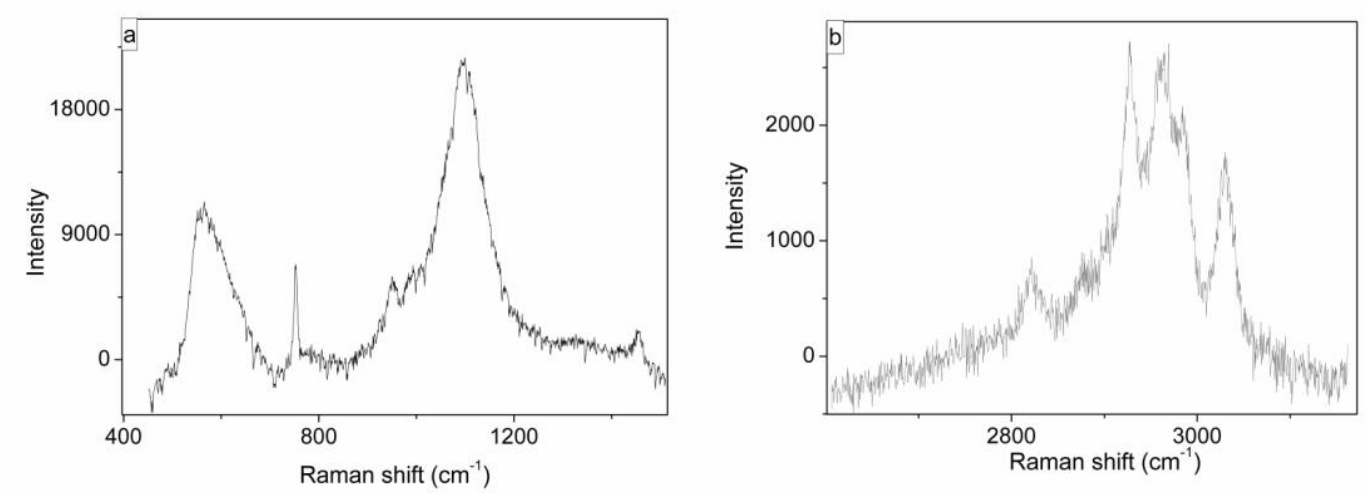

Figure 1. Raman spectra of modified-polyacrylic acid. The amine bond of polyacrylic acid and octylamine can be observed in this measurement.

\subsection{TEM and EDX}

Morphology, size, size distribution, and shape of the iron oxide NPs were acquired by means of transmission electron microscopy (HRTEM, 200keV JOEL ARM-200F). The samples were prepared by placing a suspension drop on a carbon coated copper grid followed by evaporation of the extent of the liquid under ambient conditions. The diameter of iron oxide NPs was evaluated by measuring at least 100 particles. Figure 2 shows HRTEM image of the NPs coated with modified polyacrylic acid that are well separated in the buffer solution. The mean size of the particles is $4 \mathrm{~nm}$ in diameter (Table 1). The expected thickness of the modified shell is $170 \mathrm{~nm}$ in diameter (Dynamic Light Scattering measurment, data not shown). 

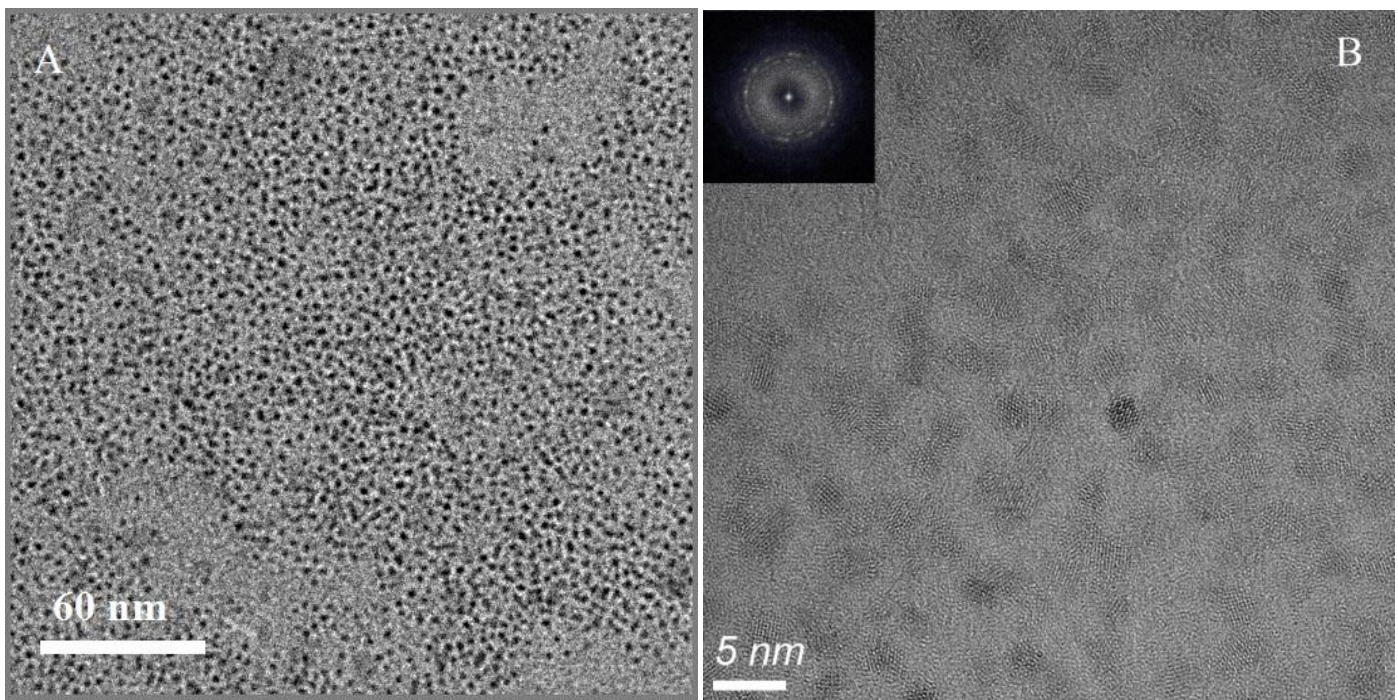

Figure 2. HRTEM images of monodisperse $4 \mathrm{~nm}$ iron oxide NPs coated with modifiedpolyacrylic acid and deposited from PBS solution (A and B). Inset in B: electron diffraction pattern demonstrating the crystallinity of randomly oriented particles. The main reflex distance corresponds to a interplanar distance of $0.298 \mathrm{~nm}$

The chemical composition of MNPs was investigated by energy-dispersive X-ray (EDX) spectroscopy in TEM. Beside iron and oxygen also the elements of PBS, i.e. phosphate, potassium and sodium were found (Figure 3). EDX analysis showed the weight percentage of oxygen and iron on the surface of iron oxide nanoparticles was respectively $26.8 \%$ and $73.2 \%$. The EDX data didn't display only the peaks from $\mathrm{Fe}$ and $\mathrm{O}$ atoms, but also potassium and sodium which are components of a phosphate buffer saline. Moreover, copper peak form base was observed.

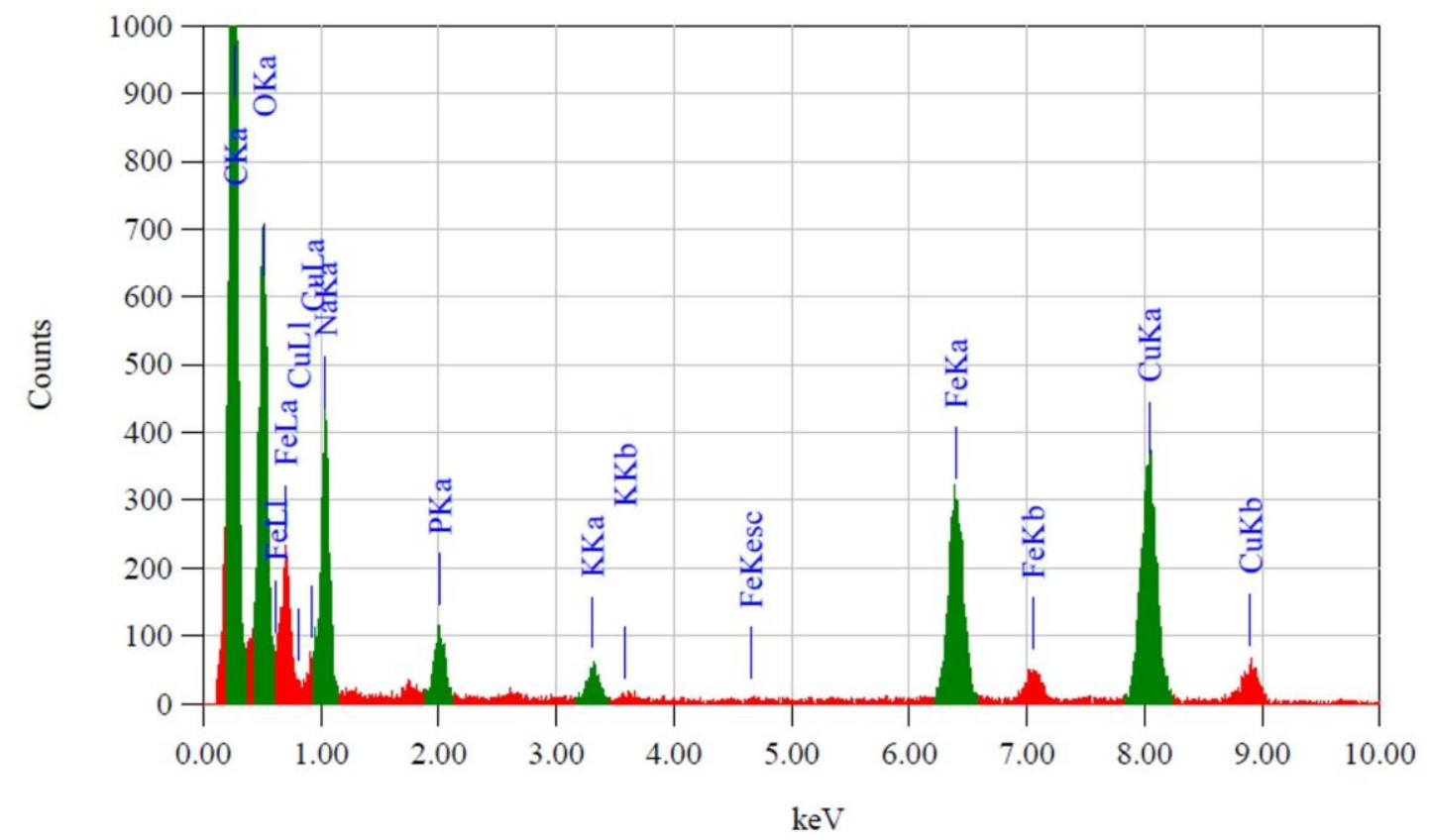

Figure 3. Energy-dispersive X-ray spectroscopy (EDX) of iron oxide NPs coated with modified-polyacrylic acid. Green peaks corresponds to Kalpha and red peaks to Kbeta. Peaks of ironoxide and phosphate buffer are present. 


\subsection{Superconducting Quantum Interference Device}

The magnetic properties were measured by a superconducting quantum interference device (SQUID, Quantum Design-MPMS XL). Subsequently the AC susceptibility was recorded to explore profoundly the nature of NPs interactions $[16,17,18]$. For a better comprehension of magnetic properties of the system, the magnetization measurements were performed by studying the temperature and field dependence. The energy barrier distribution of the samples was characterized by zero-field cooled-field cooled measurements (ZFC/FC). Firstly, the samples were cooled down to $5 \mathrm{~K}$ without an external magnetic field followed by heating up to $270 \mathrm{~K}$ in a field of $100 \mathrm{Oe}$. Then, the samples were cooled down to $5 \mathrm{~K}$ in the field (100 Oe) and the magnetization was recorded once the sample was heated to $270 \mathrm{~K}$ in the same field. For the NP (diameter $4 \mathrm{~nm}$ ) coated with modified-polyacrylic acid, the blocking temperature value could not be detected (data not shown).

Hysteresis loops were obtained at five different temperatures between $5 \mathrm{~K}$ and $270 \mathrm{~K}$. The saturation magnetization values at $5 \mathrm{~K}$ are familiar to those reported for a bulk material. The hysteresis data indicated that the saturation magnetization decreased with increasing temperature and no saturation could be observed above $5 \mathrm{~K}$ at the maximum applied field of 6000 Oe. Superparamagnetic behavior of the iron oxide was observed in the magnetization curve below $100 \mathrm{~K}$ (Figure 4), means that the superspin could rapidly reorient along the easy axis [19]. The anisotropy energy characterizes the single monodomain. Magnetic energy of a nanomagnet depends on the direction of its magnetization vector (with respect to the crystallographic directions). The directions that minimize this magnetic energy are called anisotropy directions or easy axes. The magnetic energy increases with the tilt angle between the magnetization vector and the easy directions [20].

Decreasing coercivity as a function of temperature was observed from the hysteresis loop measurements. Over the blocking temperature NPs become more stable. For all measured temperatures the coercivity field is smaller than 16 Oe.

$\mathbf{A}$

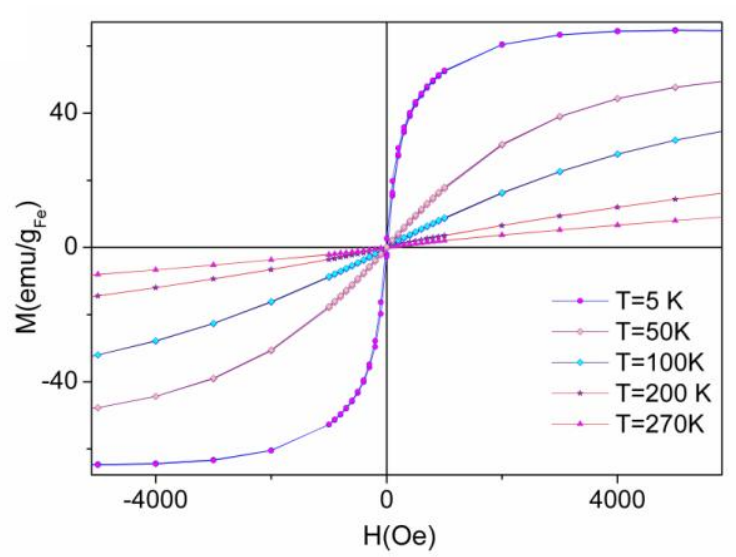

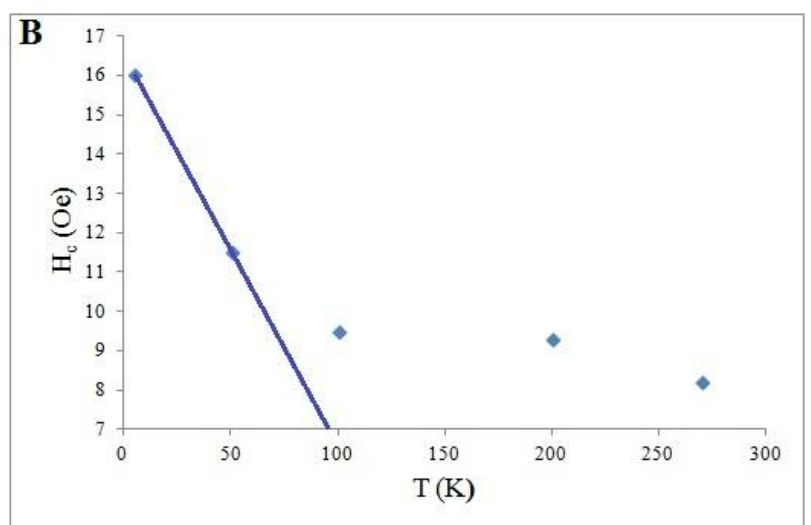

Figure 4. A: Hysteresis loops of the iron oxide NPs for temperatures between $5 \mathrm{~K}$ to $270 \mathrm{~K}$. $\mathrm{B}$ : Relation between coercivity field and temperature. The blocking temperature around $100 \mathrm{~K}$ can be estimated from extrapolated line. 
One of the interesting features of nanosize magnetic materials is the presence of a magnetic relaxation process. The process can be explained with the thermal effect and the existence of energy barriers separating local minima for different equilibrium states of the system. As a result, the system becomes blocked below the blocking temperature (TB) at which the experimental time window $(\tau)$ is equal to the relaxation time of the particles. The time window is different for AC and DC measurements. The measurement time is typically 1-100 sec for DC measurements, and is the inversion of the measurement frequency for $\mathrm{AC}$ measurements.

In order to estimate the influence of dipolar interactions the dynamic of the blocking temperature process was studied by measuring in-phase and out-of-phase susceptibility.

For single domain nanoparticles, the thermal energy barrier leads to a relaxation time $\tau$ characterized by Arrhenius' law (1):

$$
\tau(T)=\tau_{0} \exp \left(E_{A} / k_{B} T\right)
$$

where $E_{A}$ is the anisotropy energy barrier, $\tau_{0}$ is the attempt frequency and $\mathrm{k}_{\mathrm{B}}$ is the Boltzman constant. For the simplest approximation, the effective anisotropy constant can be calculated as the sum of a volume term $K_{V}$ and a surface term $K_{S}$ according to the phenomenological expression (2) [21,22]:

$$
K_{\text {eff }}=K_{V}+(6 \Phi / D) K_{S}
$$

where $K_{V}$ is the bulk anisotropy energy per volume unit, $K_{S}$ is the surface density of anisotropy energy and $6 \Phi / D$ the surface to volume ratio.

AC susceptibility measurements versus phase dependence of temperature were obtained for eight different frequencies from $10 \mathrm{~Hz}$ to $1488 \mathrm{~Hz}$. Figure 5 displays the temperature dependence of $\chi^{\prime}(\mathrm{T})$, and exhibited typical superparamagnetic behavior of the nanoparticles in the presence of a maximum (in real part of susceptibility) at the blocking temperature $T_{B}$. The $T_{B}$ value shifted towards higher temperature with increasing frequency [23,24,25]. The magnetic response becomes "frozen" below a blocking temperature, which increases with the frequency of excitation magnetic field. 


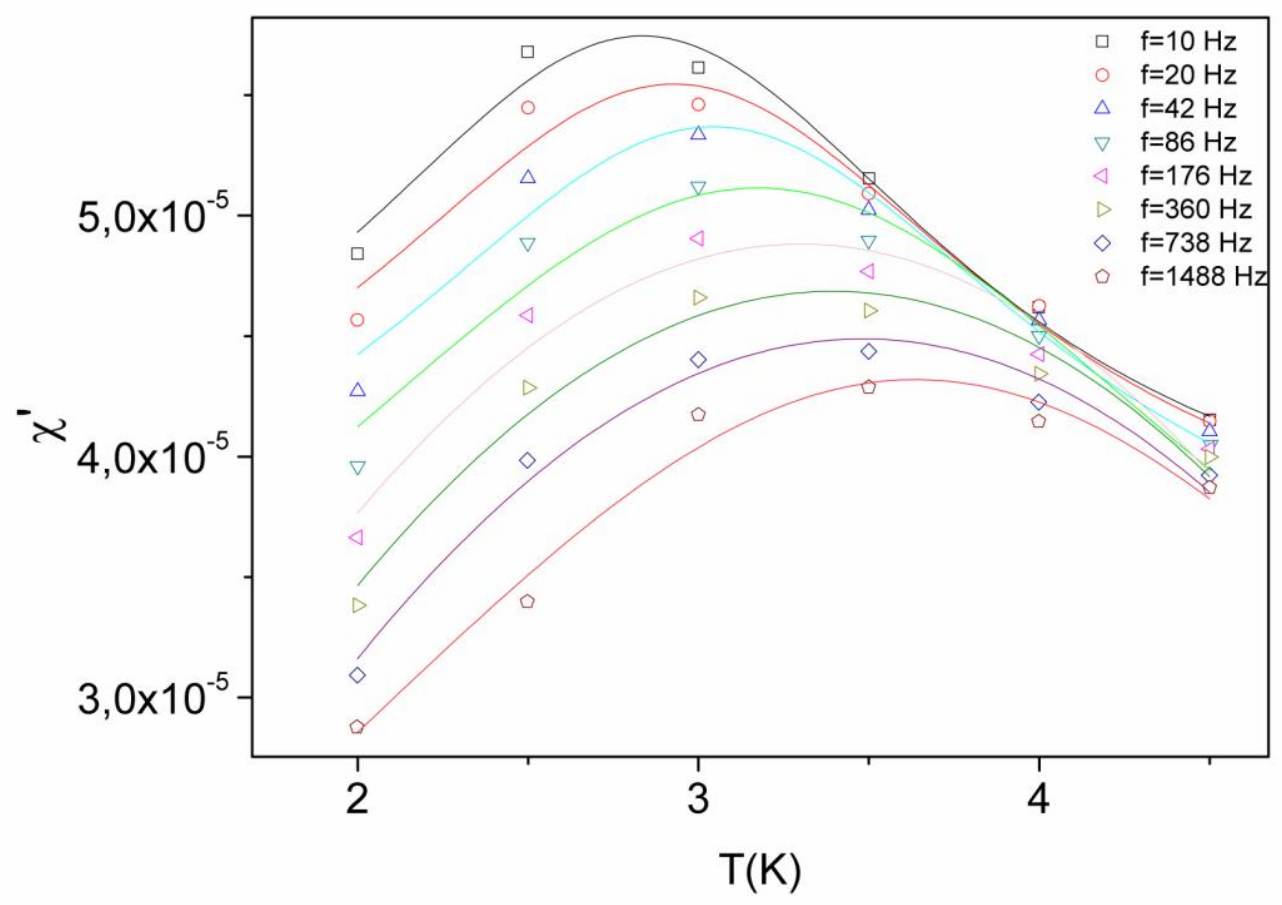

Figure 5.AC susceptibility in phase dependence of temperature. Shift of the peak maximum with increased frequency can be observed.

The dynamic response of the system was determined by measuring time $\tau$ (or frequency). For non-interacting particles the relaxation time followed an Arrhenius law (eq.1). The plot of $\ln (\tau)=\mathrm{f}\left(\mathrm{T}^{-1}\right)$ is a straight line, from which the energy barrier $\mathrm{E}_{\mathrm{A}}=65 \mathrm{~K}$ was acquired, (Figure 6). The solid line is a fit from eq. 1. The value of this parameter indicated that the dipolar interaction between nanoparticles was very weak [22], which in generally observed in well separated non-interacting particle-assemblies.

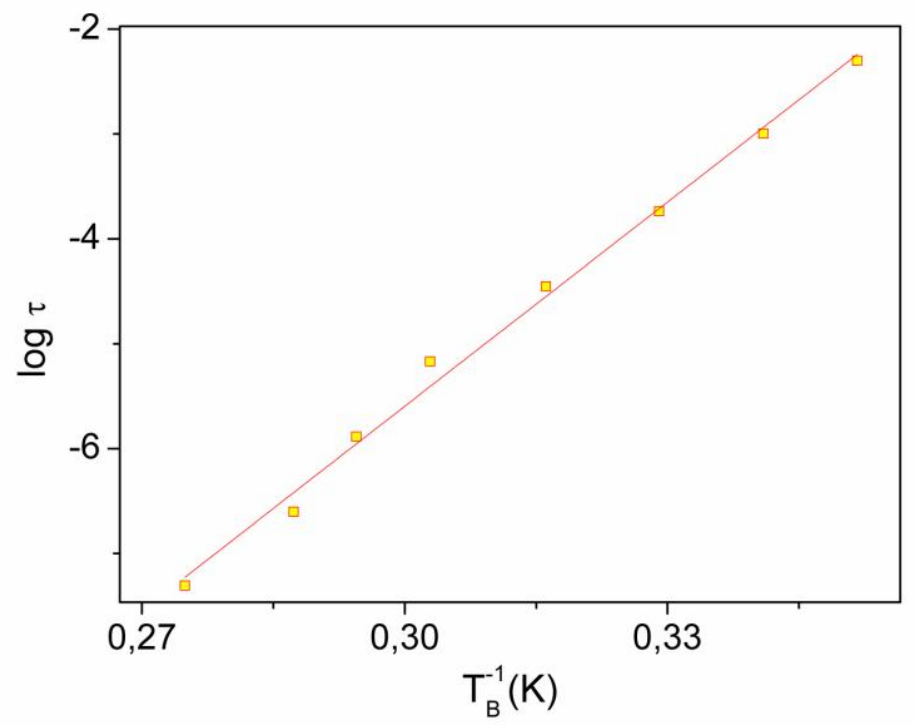

Figure 6. Arrhenius plot of the relaxation time $\tau$ versus reversed temperature $\mathrm{T}^{-1}$. The solid line is fit from eq. 1. Energy barrier $\mathrm{E}_{\mathrm{A}}=65 \mathrm{~K}$ was acquired. 
Magnetic anisotropy is considered to be one of the key factors controlling the properties of magnetic nanoparticles. From fitting data process using eq 2 and information obtained from TEM measurements the value of the effective anisotropy $K_{\text {eff }}$ was calculated and appeared to be $5 \times 10^{6} \mathrm{erg} / \mathrm{cm}^{3}$. The resulting anisotropy of nanoparticles coated by modified-polyacrylic acid is an order of magnitude larger than for the bulk material $\left(\mathrm{K}=1,35 \times 10^{5} \mathrm{erg} / \mathrm{cm}^{3}\right)$ and close enough to the value of hydrophobic iron oxide nanoparticles of similar size coated by oleic acid [22]. This enhancement is typical for magnetic nanoparticles due to contribution of the surface moment.

\section{Conclusion}

In this work we presented the analysis of magnetic properties of non-interacting iron oxide nanoparticles. Above a temperature of $5 \mathrm{~K}$ the nanoparticles are superparamagnetic. Above around $100 \mathrm{~K}$ the nanoparticles show paramagnetic behavior. The stability of the nanoparticles in physiological solution was improved by a coating with modified polyacrylic acid. The separation of the individual particles was confirmed by HRTEM and magnetic measurements. Besides, the samples showed the typical behavior for single domain particles, i.e. no coercivity. The data obtained for the real part of the susceptibility showed a temperature increase as a function of increasing frequency, which is typical for superparamagnetic nanoparticles. Using the Arrhenius model we found out that the energy barrier of the system is $65 \mathrm{~K}$. This value of the energy barrier was lower than the data obtained for the iron oxide nanoparticles.

\section{Acknowledgements}

This work was supported by UMO-2012/06/A/ST4/00373 grant from National Science Centre (Poland). We also would like to thank Karol Zaleski and Jaroslaw Mazuryk for discussions concerning the research reported here.

\section{REFERENCES}

[1] A. G. Roca, M. P. Morales, K. O’Grady and C. J. Serna, Nanotechnology 17, 2783- 2788 (2006).

[2] T. Hyeon, S. Seong Lee, J. Park, Y. Chung, and Hyon Bin Na, J. Am. Chem. Soc. 123, 12798-12801 (2001).

[3] J. Park, K. An, Y. Hwang, J. Park, H. Noh, J.Kim, J. Park, N.Hwang and T. Hyeon, Nat. Mater. 3, 891-895 (2004).

[4] V. Salgueirino-Maceira, M.A. Correa-Duarte and M. Farle, Small 1,1073-1076 (2005)

[5] M.G. Soliman, B. Pelaz, W.J. Parak and P. del Pino, Chem Mater 27, 990-997 (2015) 
[6] B. Freund, U.I. Tromsdorf, O.T. Bruns, M. Heine, A. Giemsa, A. Bartelt, S.C. Salmen, N. Raabe, J. Heeren, H. Ittrich, R. Reimer, H. Hohenberg, U. Schumacher, H. Weller and P. Nielsen, ACS Nano 6, 7318-7325 (2012)

[7] D. Ling, N. Lee and T. Hyeon, Acc Chem Res 48, 1276-1285 (2015)

[8] R. Hufschmid, H. Arami, R. M. Ferguson,a M. Gonzales, E. Teeman, L. N. Brush, N D. Browning and K. M. Krishnan , Nanoscale (2015) [Epub ahead of print]

[9] P. Perez PhD thesis, Center of Advanced European Studies and Research, Bonn (2008)

[10] X. Tenga and H. Yang, J. Mater. Chem. 14, 774- 7799, (2004)

[11] G.F. Goya, T.S. Berquó, F.C. Fonseca and MP Morales J. Appl. Phys. 94, 3520-528 (2003).

[12] N. Insin, PhD thesis, Massachusetts Institute of Technology, Boston (2011).

[13] Y. Kim, W. Kim, H. Yoo and S. Koo Shin, Bioconjugate Chem. 21, 1305-1311 (2012).

[14] C. Murli and Y. Song J. Phys. Chem. B 114, 9744-9750 (2010)

[15] M. Todica, C.V. Pop, L. Udrescu and S. Traian, Chin. Phys. Lett. 28, 12 (2011)

[16] C.E. Botez, J. L. Morris, M. P. Eastman, Chem. Phys. 403, 89-93 (2012).

[17] M. El-Hilo, K. O’Grady and R.W. Chartell, J. Magn. Magn. Mater. 114, 295-306 (1992).

[18] J. L. Dormann, L. Bessais and D. Fiorani, J. Phys. C: Solid State Phys. 21, 2015-2034 (1987).

[19] R. H. Kodama, , J. Magn. Magn. Mater. 200, 359-372 (1999).

[20] Sophie Laurent, Delphine Forge, Marc Port, Alain Roch, Caroline Robic, Luce Vander Elst, and Robert N. Muller, Chem. Rev. 108, 2064-2110 (2005)

[21] L.F. Gamarra , G.E.S. Brito, W.M. Pontuschka, E. Amaro, A.H.C. Parma, G.F. Goya, J. Magn. Magn. Mater. 289, 439-441 (2005).

[22] A. Demortiere, P. Panissod, B. P. Pichon, G. Pourroy, D. Guillon, B. Donnio and S. Begin-Colin, Nanoscale 3, 225-232 (2011).

[23] A. Zelenakova, V. Zelenak, J. Kovac, Acta ilectrotech. inform. 10, 39-42 (2010).

[24] J. L. Dormann, D. Fiorani, R. Cherkaoui, E. Tronc, F. Lucari, F. D’Orazio, L. Spinu, M. Nogues, H. Kachkachi, J.P. Jolivet, J. Magn. Magn. Mater. 203, 23-27 (1999).

[25] P. Poddar, T. Telem - Shafir, T. Fried and G. Markovich, Phys. Rev. B 66, 060403-1060403-4 (2002).

Table 1. Size distribution of nanoparticles 


\begin{tabular}{|l|l|}
\hline Number of nanoparticles & Mean size (nm) \\
\hline 270 & 2 \\
\hline 2250 & 3 \\
\hline 2470 & 4 \\
\hline 100 & 5 \\
\hline
\end{tabular}

